$\mathrm{MPI} / \mathrm{PhT} / 98-72$

hep-ph/9809503

September 1998

\title{
Neutrino oscillations in space within a solvable model
}

\author{
Ara Ioannisian* and Apostolos Pilaftsis ${ }^{\dagger}$ \\ Max-Planck-Institut für Physik (Werner-Heisenberg-Institut), \\ Föhringer Ring 6, 80805 Munich, Germany
}

\begin{abstract}
We study neutrino oscillations in space within a realistic model in which both the source and the target are considered to be stationary having Gaussianform localizations. The model admits an exact analytic solution in field theory which may be expressed in terms of complementary error functions, thereby allowing for a quantitative discussion of quantum-mechanical (coherent) versus statistical (incoherent) uncertainties. The solvable model provides an insightful framework in addressing questions related to propagation and oscillation of neutrinos that may not be attainable by the existing approaches. We find a novel form of plane-wave behaviour of neutrino oscillations if the localization spread of the source and target states due to quantum mechanics is of macroscopic size but much smaller than neutrinos' oscillation length. Finally, we discuss the limits on the coherence length of neutrino oscillations and find that they mainly arise from uncertainties of statistical origin.
\end{abstract}

PACS no.: 14.60.Pq

To appear in Physical Review D

\footnotetext{
*On leave of absence from Yerevan Physics Institute, Alikhanian Br. 2, 375036, Yerevan, Armenia †Present address: Theory Division, CERN, CH-1211 Geneva 23, Switzerland
} 
It was thirty years ago when Pontecorvo suggested [1] that if the known light neutrinos $\nu_{e}, \nu_{\mu}$ and $\nu_{\tau}$ have non-zero masses and mixings, they may oscillate to each other in the very much the same way as the $K^{0}$ and $\bar{K}^{0}$ mesons do [2]. This mechanism has also been invoked to explain the energy deficit between the neutrinos produced in the sun or earth's atmosphere and those detected on earth's ground [3]. In particular, according to the recent results of the Super-Kamiokande collaboration [4, the experimental data may comfortably be explained by naively assuming $\nu_{\mu}$-to- $\nu_{\tau}$ oscillations, when compared to the non-oscillation scenario of the Standard Model. For neutrino energies $E$ much larger than their respective masses $m_{i}$ (with $i=1,2,3$ ), the classical formula [1] governing the probability of $\nu_{\alpha}$-to- $\nu_{\beta}$ oscillations as a function of the distance $l$ from the source reads

$$
P_{\alpha \rightarrow \beta}(l)=\sum_{j=1}^{3}\left|V_{\alpha j}\right|^{2}\left|V_{\beta j}\right|^{2}+2 \sum_{j>k}^{3} \Re e\left(V_{\alpha j} V_{\beta j}^{*} V_{\alpha k}^{*} V_{\beta k}\right) \cos \left(\frac{\left(m_{j}^{2}-m_{k}^{2}\right) l}{2 E}\right),
$$

where $V_{\alpha j}$ is a leptonic mixing matrix analogous to the Cabbibo-Kobayashi-Maskawa mixing matrix of the quark sector. For simplicity, we have also neglected possible effects of $\mathrm{CP}$ violation in Eq. (1). Up to now, many authors [5 [17] have discussed the conditions under which the above naive cosine formula of oscillations between neutrinos or other particles may or may not be valid. In a large number of papers [5,6, 6, 10, 13, 17], the derivation of Eq. (1) has been based on using neutrino wave packets. Within other more field-theoretically oriented approaches [7, 11, 12, 14], neutrinos are treated as virtual intermediate states which are produced by some initial states and are only observed through their interaction with the target state in the detector. The latter considerations may also be related to those of the old-fashioned S-matrix approach [2,18]. However, in all different treatments present in the literature, many approximations were necessary to arrive at the known formula (1) of oscillations.

In this paper, we shall study particle oscillations in space within a model in which both the source and target states are taken to be stationary having Gaussian-type broadenings. For our illustrations, we first consider the oscillation of two scalar particles whose dynamics may well be determined by the relevant propagators at the second order of electroweak interactions. The discussion is then extended to the case of fermions, i.e., that of neutrinos. Since we assume a Gaussian-form localization for the production and detection interactions, the model admits an exact analytic solution within the framework of field theory and hence different sorts of approximations can directly be controlled for their validity. Thus, the solvable model offers an important insight into the profound issue of particle oscillations, thereby complementing related approaches discussed in [7,11,12,14]. In particular, we find that the oscillating pattern of neutrinos depends crucially on the coherent broadening of production and detection points. As long as the coherent broadening effects lie within the microscopic range, one has the usual picture of particle propagation through spherical waves 
in the three-dimensional space, as the particles are emitted from a well-localized source, e.g., neutrinos from the sun. If such a broadening, however, happens to be of mesoscopic size but still is much smaller than the oscillation length of particles, then their propagation proceeds via plane waves and their oscillation length depends explicitly on the three momentum direction of the initial states at the source.

Let us consider the $2 \rightarrow 2$ scattering process with two scalar particles as intermediate states which may oscillate to one another. The production and detection of the intermediate scalars occurs through some asymptotic states at the space points $\bar{x}$ and $\bar{y}$, respectively. For thermal-source situations under study [16], the time elapsed between production and detection cannot be measured directly. Therefore, it is legitimate to assume that to a rather good approximation, the interactions taking place in the source and target are stationary. The two points $\bar{x}$ and $\bar{y}$ are then macroscopically separated by a distance $l=|\bar{x}-\bar{y}|$. For example, for solar neutrinos $l$ is the distance between sun and earth, for atmospheric neutrinos $l$ is the distance between earth's atmosphere and detector at the ground, etc. Furthermore, we take the spatial localizations of the interactions at the source and target to be Gaussian functions peaked at $\bar{x}$ and $\bar{y}$ with dispersions of cubic form, i.e., $\delta x^{i}=\delta x$ and $\delta y^{i}=\delta y$.

With the above considerations, the amplitude for $\alpha \rightarrow \beta$ transition is given by

$$
\begin{aligned}
\mathcal{T}_{\beta \alpha}(k, \bar{y}, \delta y ; p, \bar{x}, \delta x) & =\widetilde{\mathcal{N}} \int d^{4} x d^{4} y e^{-(\vec{x}-\bar{x})^{2} / \delta x^{2}} e^{-(\vec{y}-\bar{y})^{2} / \delta y^{2}} e^{-i p x+i k y} \\
& \times \sum_{j=1}^{2} O_{j}^{\alpha \beta} \int \frac{d^{4} q}{(2 \pi)^{4}} \frac{e^{i q(x-y)}}{q^{2}-m_{j}^{2}+i \varepsilon}
\end{aligned}
$$

where $p=p_{1}+p_{2}$ and $k=k_{1}+k_{2}$ are the total momenta of the initial and final states, respectively. The overall proportionality factor $\widetilde{\mathcal{N}}$ contains coupling constants and other irrelevant multiplicative terms; it drops out after the probability rate is normalized. Furthermore,

$$
O_{j}^{\alpha \beta}=O_{\alpha j} O_{\beta j}
$$

is the usual combination of elements of the mixing matrix $O$. The $2 \times 2$ orthogonal matrix $O$ diagonalizes the mass matrix of the two-scalar system, and relates the weak to the mass eigenstates. Obviously, $\sum_{j} O_{j}^{\alpha \beta}=\delta_{\alpha \beta}$. Here, we should also remark that Lorentz invariance of the amplitude (2) is not manifest; it is broken by the space-dependent Gaussian functions. However, one can always recover the manifest form of Lorentz invariance by considering instead the Lorentz-invariant exponent $\delta k_{\mu}(x-\bar{x})^{\mu}$ for the Gaussian function with $\delta k_{\mu}=$ $\left(1 / \delta x^{0}, 1 / \delta x^{i}\right)$ and likewise for the $y$ coordinate. The stationarity condition implies that $\delta x^{0} \gg \delta x^{i}$ and $\delta y^{0} \gg \delta y^{i}$. For our inertial frame of particle production and detection, we take $\delta x^{0}, \delta y^{0} \rightarrow \infty$.

Since the interactions at $\bar{x}$ and $\bar{y}$ do not display any time dependence in the stationary 
limit discussed above, the integration over the times $x^{0}$ and $y^{0}$ can now easily be performed. The transition amplitude $\mathcal{T}_{\beta \alpha}$ then takes the form

$$
\begin{aligned}
\mathcal{T}_{\beta \alpha}(k, \bar{y}, \delta y ; p, \bar{x}, \delta x) & =\widetilde{\mathcal{N}} \delta\left(k^{0}-p^{0}\right) \int d^{3} \vec{x} d^{3} \vec{y} e^{-(\vec{x}-\bar{x})^{2} / \delta x^{2}} e^{-(\vec{y}-\bar{y})^{2} / \delta y^{2}} e^{i \vec{p} \vec{x}-i \vec{k} \vec{y}} \\
& \times \sum_{j=1}^{2} O_{j}^{\alpha \beta} \int \frac{d^{3} \vec{q}}{(2 \pi)^{3}} \frac{e^{-i \vec{q}(\vec{x}-\vec{y})}}{q_{j}^{2}-|\vec{q}|^{2}+i \varepsilon},
\end{aligned}
$$

where $q_{j}^{2}=E^{2}-m_{j}^{2}$. The result of the time integrations is that energy conservation holds strictly both at production and detection vertices, i.e., $k^{0}=p^{0}=E$, as can readily be seen from the delta function on the RHS of Eq. (4).

It is now important to notice that under spatial displacements, e.g., $\bar{x} \rightarrow \bar{x}+\vec{a}$ and $\bar{y} \rightarrow \bar{y}+\vec{a}, \mathcal{T}_{\beta \alpha}(\bar{x}, \bar{y})$ transforms into $e^{i \vec{a}(\vec{k}-\vec{p})} \mathcal{T}_{\beta \alpha}(\bar{x}, \bar{y})$. In other words, the amplitude is frame-independent up to an unobservable phase $\vec{a}(\vec{k}-\vec{p})$. Employing this fact, it proves convenient to redefine the amplitude into a manifestly frame-independent form

$$
\begin{aligned}
\mathcal{T}_{\beta \alpha}(k, p, \vec{l}, \delta y, \delta x) & \rightarrow e^{-i \vec{p} \hat{x}+i \vec{k} \bar{y}} \mathcal{T}_{\beta \alpha}(k, \bar{y}, \delta y ; p, \bar{x}, \delta x) \\
& =\widetilde{\mathcal{N}} \delta\left(k^{0}-p^{0}\right) \int d^{3} \vec{x} d^{3} \vec{y} e^{-\vec{x}^{2} / \delta x^{2}} e^{-\vec{y}^{2} / \delta y^{2}} e^{i \vec{p} \vec{x}-i \vec{k} \vec{y}} \\
& \times \sum_{j=1}^{2} O_{j}^{\alpha \beta} \int \frac{d^{3} \vec{q}}{(2 \pi)^{3}} \frac{e^{-i \vec{q}(\vec{x}-\vec{y}-\vec{l})}}{q_{j}^{2}-|\vec{q}|^{2}+i \varepsilon},
\end{aligned}
$$

with $\vec{l}=\bar{y}-\bar{x}$. It is now not difficult to carry out the Gaussian integrals over $\vec{x}$ and $\vec{y}$ (see also Eq. (A.1) in Appendix A). In this way, we obtain

$$
\mathcal{T}_{\beta \alpha}(k, p, \vec{l}, \delta y, \delta x)=\mathcal{N} \delta\left(k^{0}-p^{0}\right) \sum_{j=1}^{2} O_{j}^{\alpha \beta} \int \frac{d^{3} \vec{q}}{(2 \pi)^{3}} \frac{e^{-\delta x^{2}(\vec{p}-\vec{q})^{2} / 4} e^{-\delta y^{2}(\vec{k}-\vec{q})^{2} / 4} e^{i \vec{q} \vec{l}}}{q_{j}^{2}-|\vec{q}|^{2}+i \varepsilon},
$$

with $\mathcal{N}=\pi^{3} \delta x^{3} \delta y^{3} \widetilde{N}$. In Appendix A we show that the very last three-dimensional integral over $\vec{q}$ in Eq. (6) can be solved exactly within a class of error functions. Taking this very last fact into consideration, we arrive at the following analytic expression:

$$
\begin{gathered}
\mathcal{T}_{\beta \alpha}(k, p, \vec{l}, \delta y, \delta x)=-\mathcal{N} \delta\left(k^{0}-p^{0}\right) \frac{1}{8 \pi|\vec{L}|} \sum_{j=1}^{2} O_{j}^{\alpha \beta} e^{-\frac{1}{4} \delta x^{2}\left(|\vec{p}|^{2}+q_{j}^{2}\right)-\frac{1}{4} \delta y^{2}\left(|\vec{k}|^{2}+q_{j}^{2}\right)} \\
\times\left[e^{i q_{j}|\vec{L}|} \operatorname{Erfc}\left(-\frac{i}{2} \delta l q_{j}-\frac{|\vec{L}|}{\delta l}\right)-e^{-i q_{j}|\vec{L}|} \operatorname{Erfc}\left(-\frac{i}{2} \delta l q_{j}+\frac{|\vec{L}|}{\delta l}\right)\right]
\end{gathered}
$$

where $\operatorname{Erfc}(z)$ is the complementary error function defined in the appendix, $\delta l^{2}=\delta x^{2}+\delta y^{2}$ and

$$
\vec{L}=\vec{l}-\frac{i}{2} \delta x^{2} \vec{p}-\frac{i}{2} \delta y^{2} \vec{k}
$$

The norm of the complex vector $\vec{L}$ is understood to act only on the vectorial space, i.e., $|\vec{L}| \equiv \sqrt{\vec{L}^{2}}$. Equation (17) is the major result of this paper, from which any behaviour of neutrino oscillations in space for different kinematic conditions may directly be inferred. 
On physical grounds, one generally expects that $\vec{k}-\vec{p}=\mathcal{O}(1 / \delta x, 1 / \delta y)$. Therefore, without sacrificing any of the physical features of particle oscillations we wish to study, we can explicitly impose a kind of three-momentum conservation for the initial and final states of the scattering in Eq. (17), i.e., $\vec{k}=\vec{p}$ [18,9]. This leaves the three-momentum of the oscillating particles still unspecified. In fact, the very same result would have been obtained, if we had started with a Gaussian interaction of the form $\exp \left[-(\vec{x}-\vec{y}-\vec{l})^{2} / \delta l^{2}\right]$, with $\delta l^{2}=\delta x^{2}+\delta y^{2}$ and integrated out first the frame-dependent components $(\vec{x}+\vec{y}) / 2$. However, we should stress that our conclusions would not change even if we considered the complete but more lengthy expression (7). Under these assumptions, the probability amplitude simplifies to

$$
\begin{aligned}
& \mathcal{T}_{\beta \alpha}(k, \vec{l}, \delta l)=-\mathcal{N} \delta^{(4)}(k-p) \frac{1}{8 \pi\left|\vec{l}-\frac{i}{2} \delta l^{2} \vec{k}\right|} \sum_{j=1}^{2} O_{j}^{\alpha \beta} e^{-\frac{1}{4} \delta l^{2}\left(|\vec{k}|^{2}+q_{j}^{2}\right)}\left[e^{i q_{j}\left|\vec{l}-\frac{i}{2} \delta l^{2} \vec{k}\right|}\right. \\
& \left.\quad \times \operatorname{Erfc}\left(-\frac{i}{2} \delta l q_{j}-\frac{\left|\vec{l}-\frac{i}{2} \delta l^{2} \vec{k}\right|}{\delta l}\right)-e^{-i q_{j}\left|\vec{l}-\frac{i}{2} \delta l^{2} \vec{k}\right|} \operatorname{Erfc}\left(-\frac{i}{2} \delta l q_{j}+\frac{\left|\vec{l}-\frac{i}{2} \delta l^{2} \vec{k}\right|}{\delta l}\right)\right] .
\end{aligned}
$$

Notice that $\mathcal{N}$ must be redefined accordingly for reasons of dimensionality. We can now determine the probability for the state $\alpha$ produced at a distant well-localized area, e.g., the sun, to observe the state $\beta$ at a macroscopic distance $l$. The normalized probability is given by

$$
\frac{d}{d \Gamma} P_{\alpha \rightarrow \beta}(k, l, \delta l)=\frac{\left|\mathcal{T}_{\beta \alpha}(k, l, \delta l)\right|^{2}}{\sum_{\beta} \int d \Gamma\left|\mathcal{T}_{\beta \alpha}(k, l, \delta l)\right|^{2}},
$$

where $\Gamma$ denotes the phase space of the final states. In Eq. (10), we have not yet included uncertainties of statistical origin which can be added incoherently (we defer the discussion to the end of the paper). In the following, we shall focus our attention on coherent quantummechanical uncertainties.

It is very instructive to discuss the following two limiting cases: (i) $\delta l^{2}|\vec{k}| \ll l$ and (ii) $\delta l^{2}|\vec{k}| \gg l$. Let us first consider case (i). We can expand the norm of the complex vector in Eq. (9) in terms of $\delta l^{2}|\vec{k}|$, i.e.

$$
\left|\vec{l}-\frac{i}{2} \delta l^{2} \vec{k}\right|=l-\frac{i}{2} \delta l^{2} \frac{\vec{k} \vec{l}}{l}+\mathcal{O}\left(\frac{\delta l^{4}|\vec{k}|^{2}}{l^{2}}\right) .
$$

To leading order, the transition amplitude then takes on the known oscillatory form

$$
\mathcal{T}_{\beta \alpha}(k, \vec{l}, \delta l)=-\mathcal{N} \delta^{(4)}(k-p) \frac{1}{4 \pi l} \sum_{j=1}^{2} O_{j}^{\alpha \beta} e^{-\frac{1}{4} \delta l^{2}\left(\vec{k}-q_{j} \vec{l} / l\right)^{2}} e^{i q_{j} l}+\mathcal{O}\left(\frac{\delta l^{4}|\vec{k}|^{2}}{l^{2}}\right)
$$

The $\vec{k}$-dependent exponential factor in Eq. (12) controls the flow of the three-momentum between the asymptotic states and the intermediate particles, i.e., that of neutrinos. In fact, the three-momentum is conserved in the production and detection vertices up to an 
error of order $1 / \delta l$. In the limit $\delta l \rightarrow 0$, any information about the three-momentum of the oscillating system is completely lost, e.g., the total three-momentum $|\vec{k}|$ of the detecting particles can take any possible value. However, in experiments the distribution over $\vec{k}$ can in principle be measured, so physically $\delta l$ may be small but not zero. How small or how big $\delta l$ could be is a puzzling issue that will be discussed below.

After integrating over the three-momentum phase space $\Gamma$ in Eq. (10), we find the wellknown formula of particle oscillations

$$
P_{\alpha \rightarrow \beta}(E, l, \delta l)=\left|\sum_{j=1}^{2} O_{j}^{\alpha \beta} e^{i q_{j} l}\right|^{2}+\mathcal{O}\left(\delta l\left(q_{1}-q_{2}\right), \frac{\delta l^{2} q_{j}}{l}\right) .
$$

The above formula is valid under the following two conditions:

$$
\begin{array}{ll}
\text { I. } & \delta l \ll L^{\mathrm{osc}}=\frac{2 \pi}{q_{1}-q_{2}}, \\
\text { II. } & \delta l^{2} q_{j} \ll l .
\end{array}
$$

The first requirement reflects the fact that the coherent uncertainty in the position $\delta l$ for the source and target states must be much smaller than the oscillation length $L^{\text {osc }}$ in order to have a non-vanishing oscillating pattern. Within the field-theoretic framework, this constraint has first been discussed in Ref. [7] and further elaborated on in [11, 12, 14, 15]. Note that a constraint analogous to Eq. (14) may also be obtained in the wave-packet treatment if $\delta l$ is to be interpreted as the size of the neutrino wave packets [0, 6, 10, 13, 16, 17]. Nevertheless, the new requirement in Eq. (15) also affects the form of particle oscillations and deserves high attention as well. In particular, it is interesting to notice that one can satisfy condition I by grossly violating condition II. To give an example, suppose that $\delta l=1 \mathrm{~cm}, \Delta m^{2}=$ $m_{1}^{2}-m_{2}^{2}=10^{-4} \mathrm{eV}^{2}$, and the energy $E=1 \mathrm{GeV}\left(1 \mathrm{GeV} \approx 5 \times 10^{13} \mathrm{~cm}^{-1}\right)$ which is typical for atmospheric neutrinos. Then, one has $L^{\text {osc }} \approx E / \Delta m^{2} \approx 10^{8} \mathrm{~cm}$, certainly much larger than $\delta l$. On the other hand, one finds that $\delta l^{2} q_{1} \approx(1 \mathrm{~cm})^{2} \times 1 \mathrm{GeV} \approx 5 \times 10^{13} \mathrm{~cm}$ is comparable to the distance between sun and earth!

To elucidate further the implications of the new condition II for particle oscillations, we shall now discuss the behaviour of the probability amplitude for the limiting case (ii) when $\delta l^{2}|\vec{k}| \gg l$. In this limit, the norm of the complex vector $\vec{l}-\frac{i}{2} \delta l^{2} \vec{k}$ may be expanded as follows:

$$
\left|\vec{l}-\frac{i}{2} \delta l^{2} \vec{k}\right|=-\frac{i}{2} \delta l^{2}|\vec{k}|+\frac{\vec{k} \vec{l}}{|\vec{k}|}+\mathcal{O}\left(\frac{l^{2}}{\delta l^{4}|\vec{k}|^{2}}\right)
$$

Substituting the last expression into Eq. (9) and employing the asymptotic expansion of $\operatorname{Erfc}(z)$ for large values of $z$ in Eq. A.15) yields

$$
\mathcal{T}_{\beta \alpha}(k, \vec{l}, \delta l)=-\mathcal{N} \delta^{(4)}(k-p) \frac{i}{2 \pi \delta l^{2}|\vec{k}|} \sum_{j=1}^{2} O_{j}^{\alpha \beta} e^{-\frac{1}{4} \delta l^{2}\left(|\vec{k}|-q_{j}\right)^{2}} e^{i q_{j} \vec{k} \vec{k} /|\vec{k}|}+\mathcal{O}\left(\frac{l^{2}}{\delta l^{4}|\vec{k}|^{2}}\right)
$$


Because of the absence of the $1 / l$ dependence of $\mathcal{T}_{\beta \alpha}$ in Eq. (17), the propagation of the intermediate states turns over to plane waves depending explicitly on the three momentum $\vec{k}$. This intriguing plane-wave behaviour of the amplitude may be attributed to the fact that non-localization effects of the source and target quantum states within a finite space volume [19] get coherently amplified for large (mesoscopic) values of $\delta l$. Geometrically, one may attempt to visualize this counter-intuitive result of the behaviour of particle propagation as follows. It appears that the intermediate states are emitted and/or detected by means of an 'antenna' of macroscopic size $\delta l^{2}|\vec{k}|$ instead of $\delta l$ that one would have naively expected. So, if an observer were close to such an 'antenna' emitting neutrinos, say, in a distance much smaller to its size, she/he would find in principle that these particles are emitted by plane waves, whereas she/he would only recover the usual form of propagation through spherical waves if she/he were at a distance much bigger than the size of the 'antenna.' Nevertheless, we still feel that a deeper understanding of this quantum-mechanical phenomenon would be very useful.

One might now worry that averaging effects due to the phase-space integration of the final states would cancel the factor proportional to $\vec{k} \vec{l}$ which appears in the oscillating part of the probability in Eq. (17). However, this is not quite true, since the square of the transition amplitude $\mathcal{T}_{\beta \alpha}(k, \vec{l}, \delta l)$ does not depend on the three-momentum difference $k_{-}=k_{1}-k_{2}$, and hence the phase-space integration over $\Gamma$ has the effect to replace simply the oscillatory factor $\vec{k} \vec{l}$ with $\overrightarrow{p l}$. To make this explicit, we note that one can always make the following variable substitutions in the phase-space integral:

$$
\begin{aligned}
\int & d^{4} k_{1} d^{4} k_{2} \delta_{+}\left(k_{1}^{2}-m_{1}^{2}\right) \delta_{+}\left(k_{2}^{2}-m_{2}^{2}\right) \delta^{(4)}(k-p)\left|\mathcal{T}_{\beta \alpha}(k, \vec{l}, \delta l)\right|^{2} \\
& =\frac{1}{2} \int d^{4} k d^{4} k_{-} \delta_{+}\left[\frac{\left(k+k_{-}\right)^{2}}{4}-m_{1}^{2}\right] \delta_{+}\left[\frac{\left(k-k_{-}\right)^{2}}{4}-m_{2}^{2}\right] \delta^{(4)}(k-p)\left|\mathcal{T}_{\beta \alpha}(k, \vec{l}, \delta l)\right|^{2} \\
& =\frac{1}{2}\left|\mathcal{T}_{\beta \alpha}(p, \vec{l}, \delta l)\right|^{2} \int d^{4} k_{-} \delta_{+}\left[\frac{\left(p+k_{-}\right)^{2}}{4}-m_{1}^{2}\right] \delta_{+}\left[\frac{\left(p-k_{-}\right)^{2}}{4}-m_{2}^{2}\right]
\end{aligned}
$$

where $\delta_{+}\left(k_{i}^{2}-m_{i}^{2}\right)=\theta\left(k_{i}^{0}\right) \delta\left(k_{i}^{2}-m_{i}^{2}\right)(i=1,2)$. The integral in the last step of Eq. (18) is just an overall normalization constant and cancels in Eq. (10). Taking this into account, the probability of particle oscillations reads

$$
P_{\alpha \rightarrow \beta}(E, \vec{p}, \vec{l}, \delta l)=\left|\sum_{j=1}^{2} O_{j}^{\alpha \beta} e^{i q_{j} \vec{p} /|\vec{p}|}\right|^{2}+\mathcal{O}\left(\delta l\left(q_{1}-q_{2}\right), \frac{l}{\delta l^{2} q_{j}}\right) .
$$

From the above formula, it is obvious that the oscillation length as seen by the detector is strongly correlated with the three-momentum $\vec{p}$ of the initial states producing the mixed particles. In principle, the two different production mechanisms as well as their predictions obtained by Eqs. (13) and (19) could be distinguished if we were able to measure the spatial dependence of the oscillation length by moving the detector around the source. In addition, 
we must assume that one somehow knows the initial momentum $\vec{p}$ of the production mechanism and controls well both coherent $(\delta y)$ and incoherent uncertainties at the detector. Even though this seems to be a rather formidable task, the actual size of $\delta l$ dictates the nature of particle propagation within the solvable model. So far, many qualitative estimates exist for $\delta l$. However, there has not been yet a rigorous method to evaluate this quantity from first principles. It is obvious that $\delta l$ depends decisively on the details of the experiment. According to [5, 13, 10, [16], the most natural choice for the scale of $\delta l$ for neutrinos coming from the sun lies between the size of nucleus, i.e. several fm's, and $10^{-7} \mathrm{~cm}$. The latter originates from effects due to thermal collisions of electrons which results in a reduction of the wave-packet size of the production system. Therefore, it is most likely to expect that solar neutrinos have a spherical-wave propagation.

We shall now turn to the case of oscillations of two fermions such as neutrinos. Our starting point is a transition amplitude analogous to Eq. (5)

$$
\begin{aligned}
\mathcal{T}_{\beta \alpha}(k, p, \vec{l}, \delta y, \delta x) & =\widetilde{\mathcal{N}} \delta\left(k^{0}-p^{0}\right) J_{f}^{\mu}\left(k_{1}\right) \bar{u}_{\beta}\left(k_{2}\right) \gamma_{\mu} P_{L} \int d^{3} \vec{x} d^{3} \vec{y} e^{-\vec{x}^{2} / \delta x^{2}} e^{-\vec{y}^{2} / \delta y^{2}} e^{i \vec{p} \vec{x}-i \vec{k} \vec{y}} \\
& \times \sum_{j=1}^{2} V_{j}^{\alpha \beta} \int \frac{d^{3} \vec{q}}{(2 \pi)^{3}} \frac{\left(\gamma^{0} E-\vec{\gamma} \vec{q}+m_{j}\right) e^{-i \vec{q}(\vec{x}-\vec{y}-\vec{l})}}{q_{j}^{2}-|\vec{q}|^{2}+i \varepsilon} u_{\alpha}\left(p_{2}\right) J_{i}^{\nu}\left(p_{1}\right) \\
& =\mathcal{N} \delta\left(k^{0}-p^{0}\right) J_{f}^{\mu}\left(k_{1}\right) \bar{u}_{\beta}\left(k_{2}\right) \gamma_{\mu} P_{L} \\
& \times \sum_{j=1}^{2} V_{j}^{\alpha \beta}\left(\gamma^{0} E-\vec{\gamma} \nabla_{\vec{l}}\right) \int \frac{d^{3} \vec{q}}{(2 \pi)^{3}} \frac{e^{-\delta x^{2}(\vec{p}-\vec{q})^{2} / 4} e^{-\delta y^{2}(\vec{k}-\vec{q})^{2} / 4} e^{i \vec{q} \vec{l}}}{q_{j}^{2}-|\vec{q}|^{2}+i \varepsilon} \\
& \times \gamma_{\nu} u_{\alpha}\left(p_{2}\right) J_{i}^{\nu}\left(p_{1}\right) .
\end{aligned}
$$

where $p=p_{1}+p_{2}, k=k_{1}+k_{2}, P_{L}=\left(1-\gamma_{5}\right) / 2, E=k^{0}=p^{0}\left(q_{j}=\sqrt{E^{2}-m_{j}^{2}}\right)$ and

$$
V_{j}^{\alpha \beta}=V_{\alpha j} V_{\beta j}^{*} .
$$

Specifically, Eq. (20) describes the amplitude probability for producing the neutrino flavour $\alpha$ at point $\vec{x}$ through the standard $V-A$ weak current interaction $J_{i}^{\nu}\left(p_{1}\right)$ and detecting the neutrino flavour $\beta$ through a similar $V-A$ weak current interaction $J_{f}^{\mu}\left(k_{1}\right)$. The neutrino flavour states $\alpha$ and $\beta$ may be identified by the charged leptons $l_{\alpha}$ and $l_{\beta}$ which accompany the neutrinos in the production and detection vertices, respectively.

The analytic form of the transition amplitude describing neutrino oscillations is given by

$$
\begin{gathered}
\mathcal{T}_{\beta \alpha}(k, p, \vec{l}, \delta y, \delta x)=-\mathcal{N} \delta\left(k^{0}-p^{0}\right) \frac{1}{8 \pi|\vec{L}|} \sum_{j=1}^{2} V_{j}^{\alpha \beta} e^{-\frac{1}{4} \delta x^{2}\left(|\vec{p}|^{2}+q_{j}^{2}\right)-\frac{1}{4} \delta y^{2}\left(|\vec{k}|^{2}+q_{j}^{2}\right)} \\
\times\left(J_{f}^{\mu}\left(k_{1}\right) \bar{u}_{\beta}\left(k_{2}\right) \gamma_{\mu} P_{L} \operatorname{E}^{0} \gamma_{\nu} u_{\alpha}\left(p_{2}\right) J_{i}^{\nu}\left(p_{1}\right)\left(e^{i q_{j}|\vec{L}|} \operatorname{Erfc} z_{-}^{(j)}-e^{-i q_{j}|\vec{L}|} \operatorname{Erfc} z_{+}^{(j)}\right)\right. \\
-J_{f}^{\mu}\left(k_{1}\right) \bar{u}_{\beta}\left(k_{2}\right) \gamma_{\mu} P_{L} \frac{\vec{\gamma} \vec{L}}{|\vec{L}|} \gamma_{\nu} u_{\alpha}\left(p_{2}\right) J_{i}^{\nu}\left(p_{1}\right)\left\{e^{i q_{j}|\vec{L}|}\left[\left(q_{j}-\frac{1}{|\vec{L}|}\right) \operatorname{Erfc} z_{-}^{(j)}-\frac{2}{\sqrt{\pi} \delta l} e^{-z_{-}^{(j) 2}}\right]\right. \\
\left.\left.-e^{-i q_{j}|\vec{L}|}\left[\left(q_{j}+\frac{1}{|\vec{L}|}\right) \operatorname{Erfc} z_{+}^{(j)}-\frac{2}{\sqrt{\pi} \delta l} e^{-z_{+}^{(j) 2}}\right]\right\}\right),
\end{gathered}
$$


where the complex vector $\vec{L}$ is defined in Eq. (8) and $z_{\mp}^{(j)}=-\frac{i}{2} \delta l^{2} q_{j} \mp|\vec{L}| / \delta l$. Apart from obvious complications due to the spinorial structure of the analytic expression (22), the theoretical predictions as well as conclusions concerning neutrino oscillations are very analogous to the scalar case discussed above, and we shall not repeat these here. Instead, we wish to make a few clarifying remarks concerning the model under discussion. Even though the Gaussian-type spatial broadenings are equivalent to considering wave-packets for the initial (production) and final (detection) system of states, our field-theoretic approach does not involve explicitly wave-packets for the propagating particles. In fact, the propagation of the quasi-virtual intermediate states in space is consistently taken into account by their respective propagators without recourse to any boundary or preparation condition for the propagating states. Since we integrate over all times, energy conservation and flavour factorization at the source is a consequence of our model rather than an input to it.

Because of the strict energy conservation discussed above both at production and detection vertices, the formulas of particle oscillations in Eqs. (13) and (19) predict an infinitely long oscillating pattern [11]. This is not an unexpected consequence of field theory. In relativistic quantum field theory, energy and three-momentum are described by commuting independent operators in the same way as time and space variables are. This fact is also manifested in the Fock-Schwinger formulation of propagators [20]. As a consequence, energy and space may in principle be measured to infinite precision, despite the spread in threemomentum and time for the source and target states. In fact, the solvable model under discussion amounts to assuming an infinite spread in time which in turn is equivalent to an infinite sharply peaked energy distribution. Within this framework, the use of on-shell kinematic conditions to predict the energy of a quantum system by its three momentum is not a rather well justified procedure. As we will see below, an upper limit on the length of particle oscillations comes from statistical (incoherent) uncertainties within the solvable model.

In addition to the coherent quantum-mechanical uncertainties discussed above, one has to worry about statistical effects due to the energy spread of the stationary source or due to source's and detector's finite sizes in space which may give rise to destructive effects on particle oscillations. Let us discuss an illustrative example of the kind. Suppose that the validity conditions for oscillations in Eqs. (14) and (15) are satisfied and the source being in a stationary situation emits neutrinos of an average energy $\bar{E}$ with dispersion $\Delta E$, described by the function

$$
\Pi(E ; \bar{E}, \Delta E)=\frac{1}{\Delta E} \theta\left(\frac{1}{2} \Delta E-|E-\bar{E}|\right) .
$$

The function $\Pi(E)$ has the shape of a double-well with width $\Delta E$ and goes over to a deltafunction in the limit $\Delta E \rightarrow 0$. Then, for non-zero values of $\delta E$, the oscillating part of the averaged probability for $\alpha \rightarrow \beta$ transition is given by 


$$
\begin{aligned}
\mathcal{P}_{\alpha \rightarrow \beta}^{\mathrm{osc}}(l) & =\int_{-\infty}^{+\infty} d E \Pi(E ; \bar{E}, \Delta E) P_{\alpha \rightarrow \beta}^{\mathrm{osc}}(E, l) \\
& \approx 2 \Re e\left(V_{1}^{a b} V_{2}^{a b *}\right) \frac{1}{\Delta E} \int_{-\Delta E / 2}^{\Delta E / 2} d x \cos \left[\frac{\Delta m^{2} l}{2 \bar{E}}\left(1-\frac{x}{\bar{E}}\right)\right] \\
& =2 \Re e\left(V_{1}^{a b} V_{2}^{a b *}\right) \frac{\bar{E}}{\Delta E} \frac{L^{\mathrm{osc}}(\bar{E})}{\pi l} \sin \left(\frac{\Delta E}{\bar{E}} \frac{\pi l}{\left.\operatorname{Losc}^{\text {osc }} \bar{E}\right)} \cos \left(\frac{2 \pi l}{\left.\operatorname{Losc}^{\text {osc }} \overline{\bar{E}}\right)}\right)\right.
\end{aligned}
$$

where we have implicitly assumed invariance of our model under CP, i.e., $\Im m\left(V_{1}^{a b} V_{2}^{a b *}\right)=0$. From the last expression in Eq. (24), we readily see that neutrinos cease to oscillate after travelling a distance

$$
l>L^{\mathrm{coh}}=\frac{\bar{E}}{\pi \Delta E} L^{\mathrm{osc}}(\bar{E})
$$

In the literature $L^{\text {coh }}$ is called coherence length of oscillations, which characterizes the maximal scale at which neutrino oscillations can still be observed (see, e.g., [10]). By analogy, one can estimate averaging effects on oscillations due to source's and detector's finite sizes $\sigma_{x}$ and $\sigma_{y}$, respectively. Going through a similar exercise, one finds for the oscillating part of the probability

$$
\mathcal{P}_{\alpha \rightarrow \beta}^{\mathrm{osc}}(l)=2 \Re e\left(V_{1}^{a b} V_{2}^{a b *}\right) \frac{L^{\mathrm{osc}}}{\pi \sigma_{l}} \sin \left(\frac{2 \pi \sigma_{l}}{L^{\mathrm{osc}}}\right) \cos \left(\frac{2 \pi l}{L^{\mathrm{osc}}}\right)
$$

where $\sigma_{l}^{2}=\sigma_{x}^{2}+\sigma_{y}^{2}$. Despite its different origin, the validity condition for neutrino oscillations, first observed by Pontecorvo in [1], is then quite analogous to Eq. (14), i.e.

$$
\sigma_{l} \ll L^{\mathrm{osc}} .
$$

As opposed to [17], we do not find any new upper bound on $L^{\text {coh }}$ within our solvable model if $\Delta E=0$ and the requirements given in Eqs. (14) and (27) hold true. In fact, in such a case neutrinos would oscillate to infinite distances. Nevertheless, in realistic situations of production and detection of asymptotic states, $\Delta E$ is different from zero, leading to a physical cut-off for $L^{\text {coh }}[1,5,5,10,16]$. For example, for solar neutrinos, the energy dispersion $\Delta E$ of the production system is of the same order of the energy thermal spread $\Delta E_{e} \sim 1$ $\mathrm{keV}$ of electrons captured by ${ }^{7} \mathrm{Be}$.

In summary, we have studied neutrino oscillations in space in a realistic model in which both the source and target states have Gaussian-form localizations. Such a model is analytically solvable in terms of complementary error functions within the perturbative framework of field theory. This enables to take under close scrutiny various approximations used in the literature to arrive at the known formula (1) of neutrino oscillations, such as the steepest descent method or the naive pole-dominance approximation. We find that the actual spacedependence of particle oscillations obtained by the solvable model (c.f., Eqs. (17), (9) and $(22))$ is very sensitive to the localization spread $\delta l$ of the production and detection states. 
If $\delta l$ is of microscopic size such that $\delta l^{2} E \ll l$, we then recover the usual picture of particle oscillations through spherical waves given in Eqs. (1) and (13). If, however, the distance $l$ between the source and the detector is such that $\delta l^{2} E \gg l$ but $\delta l \ll L^{\text {osc }}$ and $l$, neutrino oscillations occur through plane waves and their apparent oscillation length as seen by the detector is strongly correlated with the average three-momentum $\vec{p}$ of the initial particles producing neutrinos. Such a possibility may introduce ambiguities in the determination of the mass differences of neutrinos. Which of the two afore-mentioned forms of propagation dominates in different neutrino experiments and how can these be disentangled are open challenges that present and future experiments may have to face.

Acknowledgments. We wish to thank Bill Bardeen, James Bjorken, Stanley Brodsky, Darwin Chang, George Gounaris, Pasha Kabir, Wai-Yee Keung, Mikhail Marinov, Georg Raffelt and Arkady Veinshtein for discussions. Last but not least, we are thankful to Leo Stodolsky for illuminating conversations concerning the stationary condition in neutrino oscillations. AP thanks the theoretical groups of SLAC and FERMILAB for their kind hospitality, while part of the work was performed. AI acknowledges support from the Alexander von Humboldt Foundation. 


\section{APPENDIX A: THREE-DIMENSIONAL INTEGRALS}

In this appendix we shall list analytic expressions of useful three-dimensional integrals occurring in the model under discussion. For this purpose, we first give the formula pertaining to the Gaussian integration

$$
\int_{-\infty}^{\infty} d^{3} \vec{x} e^{-i \vec{a} \vec{x}} e^{-\vec{x}^{2} / \delta x^{2}}=\pi^{3 / 2} \delta x^{3} e^{-\frac{1}{4} \delta x^{2} \vec{a}^{2}}
$$

Another useful integral is the scalar propagator in three Euclidean dimensions

$$
\Delta(\vec{l})=\int \frac{d^{3} \vec{q}}{(2 \pi)^{3}} \frac{e^{i \vec{q} \vec{l}}}{q_{j}^{2}-|\vec{q}|^{2}+i \varepsilon} .
$$

The above integral can be solved exactly by virtue of Cauchy's theorem. The intermediate steps of integration may be described as follows:

$$
\begin{aligned}
\Delta(l) & =\frac{1}{4 \pi^{2}} \int_{0}^{+\infty}|\vec{q}|^{2} d|\vec{q}| \int_{-1}^{+1} d z \frac{e^{i|\vec{q}| l z}}{q_{j}^{2}-|\vec{q}|^{2}+i \varepsilon}=-\frac{i}{4 \pi^{2} l} \int_{0}^{+\infty}|\vec{q}| d|\vec{q}| \frac{e^{i|\vec{q}| l}-e^{-i|\vec{q}| l}}{q_{j}^{2}-|\vec{q}|^{2}+i \varepsilon} \\
& =-\frac{i}{4 \pi^{2} l} \int_{-\infty}^{+\infty}|\vec{q}| d|\vec{q}| \frac{e^{i|\vec{q}| l}}{q_{j}^{2}-|\vec{q}|^{2}+i \varepsilon}
\end{aligned}
$$

with $|\vec{l}|=l$. The integrand in the last expression of Eq. (A.3) has two complex poles situated at $|\vec{q}|= \pm q_{j} \pm i \varepsilon$. For the case $l>0$, we may analytically continue the contour of integration over the upper half of the complex $|\vec{q}|$ plane. Since the integration over the complex semicircle boundary vanishes, the only non-vanishing contribution to the integral (A.3) comes from the pole $q_{j}+i \varepsilon$. Thus, we find

$$
\Delta(l)=-\frac{i}{4 \pi^{2} l}(2 \pi i) \lim _{|\vec{q}| \rightarrow q_{j}}\left(|\vec{q}|-q_{j}-i \varepsilon\right) \frac{|\vec{q}| e^{i|\vec{q}| l}}{q_{j}^{2}-|\vec{q}|^{2}+i \varepsilon}=-\frac{1}{4 \pi l} e^{i q_{j} l}
$$

In the following, we shall consider the integral contained in Eq. (6). More explicitly, we have

$$
\begin{aligned}
\mathcal{M}(k, p, \vec{l}, \delta y, \delta x) & =\int \frac{d^{3} \vec{q}}{(2 \pi)^{3}} \frac{e^{-\delta x^{2}(\vec{p}-\vec{q})^{2} / 4} e^{-\delta y^{2}(\vec{k}-\vec{q})^{2} / 4} e^{i \vec{q} \vec{l}}}{q_{j}^{2}-|\vec{q}|^{2}+i \varepsilon} \\
& =\frac{e^{-\frac{1}{4} \delta x^{2}|\vec{p}|^{2}-\frac{1}{4} \delta y^{2}|\vec{k}|^{2}}}{2 \pi^{2}|\vec{L}|} \int_{0}^{+\infty}|\vec{q}| d|\vec{q}| \frac{e^{-\frac{1}{4}\left(\delta x^{2}+\delta y^{2}\right)|\vec{q}|^{2}} \sin (|\vec{q}||\vec{L}|)}{q_{j}^{2}-|\vec{q}|^{2}+i \varepsilon},
\end{aligned}
$$

where $\vec{L}$ is defined in Eq. (8). The integral (A.5) is generally convergent since the integrand falls off exponentially in the limits $|\vec{q}| \rightarrow \pm \infty$. However, the theorem by Cauchy does not apply in this case, since the integral over the complex semi-circle with infinite radius does not vanish. Therefore, we must proceed differently.

The integral we must calculate in Eq. (A.5) has the following analytic form: 


$$
I(a, b, y) \equiv \frac{1}{2 i} \int_{0}^{+\infty} d x \frac{x e^{-a^{2} x^{2}}\left(e^{i x y}-e^{-i x y}\right)}{x^{2}+b^{2}}
$$

where $a=\frac{1}{2} \sqrt{\delta x^{2}+\delta y^{2}}>0, b=-i q_{j}+\varepsilon /\left(2 q_{j}\right)(\Re e b>0)$, and $y=|\vec{L}|$ is a complex variable. Instead of considering the integral $I(a, b, y)$, we shall first calculate

$$
F(a, b, y) \equiv \frac{1}{2 i} \int_{0}^{+\infty} d x \frac{x e^{-a^{2} x^{2}} e^{i x y}}{x^{2}+b^{2}}
$$

and then obtain $I(a, b, y)$ by the obvious relation

$$
I(a, b, y)=F(a, b, y)-F(a, b,-y) .
$$

We use Schwinger's representation for the integrand in Eq. (A.7), viz.

$$
F(a, b, y)=\frac{1}{i} \lim _{R \rightarrow+\infty} e^{a^{2} b^{2}} \int_{a}^{R / b} d t t \int_{0}^{+\infty} d x x e^{-t^{2}\left(x^{2}+b^{2}\right)} e^{i x y} .
$$

In order to avoid possible singularities originating from the complex pole $\left(x^{2}+b^{2}\right)^{-1}$ of the propagator, we could perform the usual Wick rotation and regard $x$ as a pure imaginary variable. We shall no longer dwell upon this point, since it also occurs even if $a \rightarrow 0$, in which case the result may be read off from Eq. (A.4). Thus, we can formally carry out the Gaussian integration in (A.9) and rewrite $F(a, b, y)$ in the following forms:

$$
\begin{aligned}
F(a, b, y) & =-\frac{1}{2} \lim _{R \rightarrow+\infty} e^{a^{2} b^{2}} \int_{a}^{R / b} d t t e^{-t^{2} b^{2}} \frac{\partial}{\partial y} \int_{-\infty}^{+\infty} d x e^{-t^{2} x^{2}} e^{i x y} \\
& =\frac{\sqrt{\pi}}{4} \lim _{R \rightarrow+\infty} e^{a^{2} b^{2}} \int_{a}^{R / b} \frac{d t}{t^{2}} y e^{-t^{2} b^{2}-y^{2} /\left(4 t^{2}\right)} \\
& =\frac{\sqrt{\pi}}{4} \lim _{R \rightarrow+\infty} e^{a^{2} b^{2}} e^{-b y} \int_{a}^{R / b} \frac{d t}{t^{2}} y e^{-[t b-y /(2 t)]^{2}} .
\end{aligned}
$$

Making the variable substitution $z=t b-y /(2 t)$ and taking the limit $R \rightarrow+\infty$ where appropriate, we finally arrive at

$$
F(a, b, y)=\frac{\pi}{4} e^{a^{2} b^{2}} e^{-b y} \operatorname{Erfc}\left(a b-\frac{y}{2 a}\right)-\frac{b e^{a^{2} b^{2}} \sqrt{\pi}}{4} \lim _{R \rightarrow+\infty} \int_{a}^{R / b} d t e^{-t^{2} b^{2}-y^{2} /\left(4 t^{2}\right)} .
$$

In Eq. (A.11), $\operatorname{Erfc}(z)$ is the complementary error function of a complex argument defined as

$$
\operatorname{Erfc}(z) \equiv \frac{2}{\sqrt{\pi}} \int_{z}^{+\infty} d t e^{-t^{2}}=1-\frac{2}{\sqrt{\pi}} \int_{0}^{z} d t e^{-t^{2}}
$$

The last $R$-dependent term on the RHS of Eq. (A.11) is symmetric under the interchange $y \rightarrow-y$ and cancels in $I(a, b, y)$ when the difference in Eq. (A.8) is formed. The so-derived 
analytic result agrees with that of Ref. [21] for $y>0$ which has been extended here to include complex values of $y$. After restoring all the terms present in Eq. (A.5), we eventually obtain

$$
\begin{aligned}
\mathcal{M}(k, p, & \vec{l}, \delta y, \delta x)=-\frac{1}{8 \pi|\vec{L}|} e^{-\frac{1}{4} \delta x^{2}\left(|\vec{p}|^{2}+q_{j}^{2}\right)-\frac{1}{4} \delta y^{2}\left(|\vec{k}|^{2}+q_{j}^{2}\right)} \\
& \times\left[e^{i q_{j}|\vec{L}|} \operatorname{Erfc}\left(-\frac{i}{2} \delta l q_{j}-\frac{|\vec{L}|}{\delta l}\right)-e^{-i q_{j}|\vec{L}|} \operatorname{Erfc}\left(-\frac{i}{2} \delta l q_{j}+\frac{|\vec{L}|}{\delta l}\right)\right],
\end{aligned}
$$

with $\delta l^{2}=\delta x^{2}+\delta y^{2}$.

It is useful to list a few values of $\operatorname{Erfc}(z)$ for special $z$ 's as well as derive appropriate expansions of its argument which have been used extensively throughout our discussion. In particular, we have

$$
\operatorname{Erfc}(+\infty)=0, \quad \operatorname{Erfc}(0)=1, \quad \operatorname{Erfc}(-\infty)=2
$$

The asymptotic behaviour of $\operatorname{Erfc}(z)$ for $|z| \gg 1$ with $\Re z>0$ [22] may be obtained by a series expansion, viz.

$$
\begin{aligned}
\operatorname{Erfc}(z) & =\frac{e^{-z^{2}}}{\sqrt{\pi} z}\left[1+\sum_{m=1}^{\infty}(-1)^{m} \frac{1 \times 3 \times \ldots(2 m-1)}{\left(2 z^{2}\right)^{m}}\right] \\
& =\frac{e^{-z^{2}}}{\sqrt{\pi} z}\left[1+\mathcal{O}\left(\frac{1}{z^{2}}\right)\right]
\end{aligned}
$$

If $\Re z<0$, one may employ the following property:

$$
\operatorname{Erfc}(z)=2-\operatorname{Erfc}(-z)
$$

and then apply the asymptotic expansion in Eq. (A.15). By means of Eqs. (A.15) and (A.16), we find that in the limit $\delta l \rightarrow 0$, the expression in Eq. (A.13) becomes identical to that given in Eq. (A.4). Furthermore, if $\delta l^{2} q_{j}, \delta l^{2}|\vec{k}| \ll l$ with $\vec{k}=\vec{p}$, we obtain the asymptotic expression in Eq. (12), which is in agreement with results derived in [11] from different considerations. The latter constitutes another non-trivial check of the validity of our analytic results. On the other hand, if $\delta l^{2} q_{j}, \delta l^{2}|\vec{k}| \gg l$ but $\delta l \ll l$, $L^{\text {osc }}$, the transition amplitude $\mathcal{M}$ has a non-trivial dependence on the Erfc functions in Eq. (A.13) which multiply the exponential factors $\exp \left(i q_{j}|\vec{L}|\right)$ and $\exp \left(-i q_{j}|\vec{L}|\right)$. However, the term depending on $\exp \left(i q_{j}|\vec{L}|\right)$ leads to a Gaussian-like function $\exp \left[-\delta l^{2}\left(|\vec{k}|-q_{j}\right)^{2} / 4\right]$ that controls momentum conservation, whereas the term proportional to $\exp \left(-i q_{j}|\vec{L}|\right)$ turns out to be extremely suppressed by a factor $\exp \left[-(l / \delta l)^{2}\right]$. As a consequence, we find that up to an overall normalization constant $\mathcal{N}$, the amplitude $\mathcal{M}$ exhibits a plane-wave behaviour in this kinematic range (see also Eq. (17)).

Finally, it is amusing to discuss the S-matrix limit of formula (A.13). As can be seen from Eq. (6), this corresponds to the case $\delta x, \delta y \gg l$. For simplicity, we work in the $\vec{k}=\vec{p}$ 
approximation and keep only the leading term in the asymptotic expansion (A.15). After some algebra, we find for $\vec{k} \vec{l}>0$

$$
\begin{aligned}
\mathcal{M}(\vec{k}=\vec{p}, \vec{l}, \delta l) & \approx-\frac{1}{4 \pi|\vec{L}|} e^{-\frac{1}{4} \delta l^{2}\left(|\vec{k}|^{2}+q_{j}^{2}\right)} e^{i q_{j}|\vec{L}|}+\frac{1}{4 \pi^{3 / 2}} \frac{\delta l e^{-l^{2} / \delta l^{2}} e^{i \vec{k} \vec{l}}}{l^{2}+\delta l^{4}\left(q_{j}^{2}-|\vec{k}|^{2}\right) / 4-i \delta l^{2} \vec{k} \vec{l}} \\
& \approx \frac{1}{\delta l^{3} \pi^{3 / 2}}\left[-\frac{i \pi e^{i q_{j} \vec{k} \vec{k}|| \vec{k} \mid}}{|\vec{k}|} \frac{\delta l}{2 \sqrt{\pi}} e^{-\frac{1}{4} \delta l^{2}\left(|\vec{k}|-q_{j}\right)^{2}}\right. \\
& \left.+\mathcal{P} \frac{e^{i \vec{k} l}}{q_{j}^{2}-|\vec{k}|^{2}}+i \pi e^{i \vec{k} \vec{l}} \delta_{+}\left(q_{j}^{2}-|\vec{k}|^{2}\right)\right]
\end{aligned}
$$

where $\mathcal{P}$ stands for principle value. Recognizing now the fact that

$$
\delta(x)=\lim _{a \rightarrow \infty} \frac{a}{\sqrt{\pi}} e^{-a^{2} x^{2}},
$$

and noticing the cancellation of the additional delta function $\delta_{+}\left(q_{j}^{2}-|\vec{k}|^{2}\right)$ in Eq. (A.17), it is then not difficult to reproduce the known S-matrix result

$$
\mathcal{M}(\vec{k}=\vec{p}, \vec{l}, \delta l \rightarrow \infty)=\frac{e^{i \vec{k} \vec{l}}}{\delta l^{3} \pi^{3 / 2}}\left[-i \pi \delta_{+}\left(q_{j}^{2}-|\vec{k}|^{2}\right)+\mathcal{P} \frac{1}{q_{j}^{2}-|\vec{k}|^{2}}\right] .
$$

In the usual S-matrix treatment, one first considers the limit $\delta x, \delta y \rightarrow \infty$ in Eq. (6) and then integrates over the corresponding three-momentum delta functions. Up to an irrelevant normalization, the result thus obtained is identical to that of Eq. (A.19). 


\section{REFERENCES}

[1] B. Pontecorvo, Sov. Phys. JETP 26, 984 (1968); V. Gribov and B. Pontecorvo, Phys. Lett. B28, 493 (1969); S.M. Bilenky and B. Pontecorvo, Phys. Rep. 41, 225 (1978).

[2] R.G. Sachs, Ann. Phys. (N.Y.) 22, 239 (1963); for a pedagogical introduction to CP violation, see also, P.K. Kabir, "The CP Puzzle," (Academic Press Inc. Ltd, London, 1968).

[3] For recent analyses, see e.g., J.N. Bahcall, P.I. Krastev and A. Yu Smirnov, hepph/9807216; R. Barbieri, L.J. Hall, D. Smith, A. Strumia and N. Weiner, hepph/9807235; M.C. Gonzalez-Garcia, H. Nunokawa, O.L.G. Peres and J.W.F. Valle, hep-ph/9807305, and references therein.

[4] Y. Fukuda etal., Super-Kamiokande Collaboration, hep-ex/9807003.

[5] S. Nussinov, Phys. Lett. B63, 201 (1976); B. Kayser, Phys. Rev. D24, 110 (1981).

[6] C. Giunti, C.W. Kim and U.W. Lee, Phys. Rev. D44, 3635 (1991); Phys. Lett. B421, 237 (1998); C. Giunti, C.W. Kim, J.A. Lee and U.W. Lee, Phys. Rev. D48, 4310 (1993).

[7] J. Rich, Phys. Rev. D48, 4318 (1993).

[8] J. Lowe, B. Bassaleck, H. Burkhardt, A. Rusek, G.J. Stephenson Jr. and T. Goldman, Phys. Lett. B384, 288 (1996); B. Anchochea, A. Bramon, R. Muñoz-Tapia and M. Nowakowski, Phys. Lett. B389, 149 (1996).

[9] A.D. Dolgov, A.Yu. Morozov, L.B. Okun and M.G. Schepkin, Nucl. Phys. B502, 3 (1997).

[10] K. Kiers, S. Nussinov and N. Weiss, Phys. Rev. D53, 537 (1996).

[11] W. Grimus and P. Stockinger, Phys. Rev. D54, 3414 (1996); W. Grimus, S. Mohanty and P. Stockinger, hep-ph/9807442.

[12] J.E. Campagne, Phys. Lett. B400, 135 (1997).

[13] H.J. Lipkin, Phys. Lett. B348, 604 (1995); Y. Grossman and H.J. Lipkin, Phys. Rev. D55, 2760 (1997).

[14] K. Kiers and N. Weiss, Phys. Rev. D57, 3091 (1998).

[15] Yu. V. Shtanov, Phys. Rev. D57, 4418 (1998).

[16] L. Stodolsky, Phys. Rev. D58, 036006 (1998) (electronic form).

[17] C. Giunti and C.W. Kim, Phys. Rev. D58, 017301 (1998) (electronic form). 
[18] D. Iagolnitzer, J. Math. Phys. 10, 1241 (1969), and references therein.

[19] G.C. Hegerfeldt, Phys. Rev. D10, 3320 (1974); G.C. Hegerfeldt and S.N.M. Ruijsenaars, Phys. Rev. D22, 377 (1980).

[20] C. Itzykson and J.-B. Zuber, "Quantum Field Theory," (McGraw-Hill Inc., New York, 1980) p. 100.

[21] A. Erdelyi, W. Magnus, F. Oberhettinger and F.G. Tricomi, "Tables of integral transforms," (McGraw-Hill Inc., New York, 1954), Vol. 1, p. 74 (no. 26).

[22] M. Abramowitz and I.A. Stegun, "Handbook of Mathematical Functions," (Verlag Harri Deutsch, Frankfurt, 1984), p. 85 (nos. 7.1.23 and 7.1.14). 